\title{
Treatment guidelines of chronic inflammatory demyelinating polyneuropathy in China
}

\author{
Li-Ying Cui', Chuan-Qiang $\mathrm{Pu}^{2}$, Xue-Qiang $\mathrm{Hu}^{3}$ \\ ${ }^{1}$ Department of Neurology, Peking Union Medical Hospital, Chinese Academy of Medical Science, Beijing 100730, China. \\ ${ }^{2}$ Department of Neurology, Chinese PLA General Hospital, Beijing 100853, China. \\ ${ }^{3}$ Department of Neurology, the Third Affiliated Hospital of Sun Yat-Sen University, Guangzhou 510630, Guangdong, China.
}

Correspondence to: Dr. Li-Ying Cui, Department of Neurology, Peking Union Medical Hospital, Chinese Academy of Medical Science, Beijing 100730, China. E-mail: pumchcly@yahoo.com.cn

\begin{abstract}
How to cite this article: Cui LY, Pu CQ, Hu XQ. Treatment guidelines of chronic inflammatory demyelinating polyneuropathy in China. Neuroimmunol Neuroinflammation 2017;4:20-3.
\end{abstract}

Article history: Received: 25-03-2016 Accepted: 30-11-2016 Published: 20-02-2017

Chronic inflammatory demyelinating polyradiculoneuropathy, or chronic inflammatory demyelinating polyneuropathy (CIDP) is an acquired immune-mediated inflammatory disorder at the peripheral nervous system, in which the progression is chronic and also remission relapse. In most cases, it is also associated with cerebrospinal fluid (CSF) protein-cell separation. Electrophysiologically, the peripheral nerve conduction velocity decreases, blocks and characterized as discrete abnormal waveform. Pathologically, there is also multifocal demyelination of myelinated fibers, nerve endometrial edema, inflammatory cell infiltration, etc.

CIDP can be classified as classical and variant types, the latter of which is rare, including pure motor, pure sensory, distal acquired demyelinating symmetric (DADS) neuropathy, multifocal acquired demyelinating sensory and motor (MADSAM, also known as LewisSumner syndrome) neuropathy, etc. ${ }^{[1]}$

\section{CLINICAL SYMPTOMS AND CLASSIFICATION}

\section{Classical CIDP}

(1) This will onset at different age groups, and commonly found in 40-60 years old. There is no gender difference in onset rate; (2) no clear history of infection; (3) classifications: this can be classified into two sub-types: chronic progressive and remission relapsing. For young patients, there are more cases of remission relapsing subtype, who will have a better prognosis. For elderly patients, there are more cases of chronic progressive sub-type, who will have a worse prognosis; ${ }^{[2]}$ (4) clinical symptoms: the symptoms onset chronically, which progress over eight weeks; ${ }^{[3]}$ but $16 \%$ of the CIDP patients showed a subacute onset, the symptoms of which progress rapidly and reaches to a peak in 4-8 weeks. Additionally, patients are sensitive to glucocorticoid response. Patients are classified as CIDP tendency but not acute inflammatory demyelinating polyradiculoneurophy. CIDP symptoms confined to the peripheral nervous system, mainly including: $(A)$

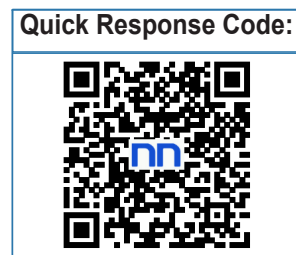


cranial nerves abnormalities: less than $10 \%$ of patients suffer from facial paralysis or opthalmoplegia. ${ }^{[2]}$ In some cases, the cranial nerves innervated bulbar muscles are compromised. In even rare cases, there will be papilledema; (B) gravis: in most cases of CIDP of classical type, patients suffer from gravis, which extend to both proximal and distal extremities; (C) sensory disturbances: most patients also suffer from numbness of the limbs or occasionally pain. There is sometimes loss of glove- or sock-like pinprick and deep sensation. In severe cases, there is also severe sensory ataxia; (D) abnormal tendon reflexes: tendon reflexes are diminished or even disappeared. In some cases, there would be reduction or loss of tendon reflexes in normal muscle; (E) autonomic dysfunction: it is expressed as orthostatic hypotension, sphincter dysfunction, arrhythmia, etc.

\section{Variant CIDP}

(1) Pure motor CIDP: about $10-11 \%$. There is only the numbness without any sensory symptoms; (2) pure sensory CIDP: about $8-17 \%$. There are only sensory symptoms, e.g. sensory ataxia, numbness and pain. However, with the extension of the course, there may be symptoms of motion expressed; (3) DADS: numbness and/or sensory impairments limited in the distal extremities. DADS is onset slower that the classical CIDP, some of which is a category of IgM monoclonal obulinemia, a subtype of monoclonal gammopathy of unknown significance (MGUS), and associated with peripheral neuropathy. Steroid therapy does not produce any therapeutic effect. In contrast, CIDP without IgM monoclonal obulinemia is sensitive to steroid therapy; (4) MADSAM: there is limb asymmetrical sensorimotor peripheral neuropathy, which is clinically similar to multifocal motor neuropathy $(\mathrm{MMN})$, but there is also evidence of sensory damages and no anti-ganglioside $\mathrm{GM}_{1}$ antibody titer. ${ }^{[4]}$

\section{ACCESSARY EXAMINATION}

\section{Electrophysiology}

In the motor nerve conduction examination, there is peripheral nerve demyelination. There is also conduction block at the entrapment site or discrete abnormal waveform, which is useful for diagnosis for demyelination. Median, ulnar, tibial and common peroneal nerves are commonly diagnosed. Results of electrophysiological tests should be consistent with the clinical symptoms. The standardized criteria of electrophysiological diagnosis includes: (1) motor nerve conduction: there is at least one of two testing motor nerves with the following abnormalities. (A) Fifty percent or more prolongation of distal latencies; (B) velocity of motor nerve conduction is $30 \%$ or above lower than the lower limit; (C) twenty percent or more prolongation of $\mathrm{F}$ wave latency than the normal upper limit. When examining the amplitude of negative phase wave, which is lower than the lower limit by $20 \%$, the F wave latency should be prolonged by $50 \%$ or more. In certain cases, there is also absence of $F$ waves; (D) partial blockade of motor nerve conduction: when comparing the proximal and distal end of the normal segments of peripheral nerves, the former amplitude of negative phase of compound muscle action potential (CMAP) decreases by $50 \%$ or more. If the reduction is less than $20 \%$, the reliability of this examination is not guaranteed; (E) discrete abnormal waveform: the duration of CMAP negative phase wave is widened for $30 \%$ or above. (2) Sensory nerve conduction: there could be delay of sensory nerve conduction and/or decrease of amplitude. (3) Electromyography with needle electrodes: although it is usually present as normal, there could be abnormal spontaneous potential, prolongation and elevation of amplitude of potential of motor units, and even loss of motor units when there is secondary axonal damage. ${ }^{[3]}$

\section{CSF}

There are about $80-90 \%$ of patients with CSF proteincell separation, which is about $0.75-2.00 \mathrm{~g} / \mathrm{L}$ (in some cases, it can be higher than $2.00 \mathrm{~g} / \mathrm{L}$ ).

\section{Biopsy of sural nerve}

In the situation that the electrophysiology test result is not consistent with the clinical symptoms and vasculitic peripheral neuropathy and hereditary neuropathy cannot be excluded, biopsy of sural nerve is necessary. The main pathological hallmarks include segmental demyelination of myelinated nerve fiber, axonal degeneration, proliferation of Schwann cell with onion-like formation, mononuclear cell infiltration, etc.

\section{DIAGNOSIS AND DIFFERENTIAL DIAGNOSIS}

\section{Diagnosis}

The current diagnosis of CIDP is in fashion of exclusion. One can be suspected as CIDP when the following criteria are fit: (1) chronic progression or remission relapse of CIDP associated symptoms over eight weeks; (2) numbness of proximal and distal extremities in different degrees in symmetrical manner; however, some are in asymmetrical pattern such as MADSAM. The reduction or loss of tendon reflexes with also depth paresthesia; (3) CSF protein-cell separation; (4) reduction and blockade of peripheral nerve conduction or discrete abnormal waveform; (5) excluded as other neuropathies; (6) improved by glucocorticoids. ${ }^{[4,5]}$ 


\section{Differential diagnosis}

(1) POEMS syndromes, or Crow-Fukase syndrome, demyelination based polyneuropathy, organomegaly of liver, spleen and lymph nodes, endocrine abnormalities such as diabetes, hypothyroidism and etc., $M$ protein (mainly IgG type with elevation) and darkening skin. Systemic multi-system examination is needed for diagnosis; (2) MMN: it is a kind of motor specific asymmetrical chronic acquired dymelinating polyneuropathy (CADP), which usually onset in adult male. MMN is onset with asymmetrical numbness of distal of upper extremities, which spreads to the proximal ends of upper extremities and lower. However, in some cases, onset could be initiated at lower extremities. Most of the affected muscle distributes as in a mononeuropathy. However, electrophysiology indicates multifocal distribution of motor transmission blockade. MMN does not highly differ from classical CIDP, but it is similar to MADSAM. The major difference of these two conditions is as followed: MMN is not with any sensory related symptoms but anti-ganglioside $\operatorname{lgM}, \mathrm{GM}_{1}$, is found in serum. Intravenous immunoglobulin, IVIg, or cyclophosphamide (CTX), but not glucocorticoid, could improve the symptoms. For MADSAM, there is not anti-ganglioside IgM in serum but glucocorticoid is efficacious in treatment; (3) cancers causing peripheral neuropathy (Paraneoplastic syndromes): it is a non-metastatic peripheral neuropathy but it could onset before, synchronized and after the carcinogenesis. It is found usually in patients at middle or elder ages, which a progressive disorder not treatable by glucocorticoid. This can be diagnosed by comprehensive examination and identification of tumor; (4) MGUS associated with peripheral neuropathy: CADP could be seen in MGUS with unknown etiological reasons, mainly IgM type. Unlike to CIDP, MGUS associated peripheral neuropathy demonstrated more sensory symptoms than motor and more significant at the distal extremities. About $50 \%$ of patients are with positive result in the test of antimyelin-associated glycoprotein antibody. This disorder cannot be efficiently treated by immunosuppressive or immunomodulatory agents, but rituximab is potent in the treatment. In some cases, the MGUS in IgG or IgA types are associated with CADP, which is with similar clinical symptoms and electrophysiology. The key diagnosis highly relies on the positive finding of $\mathrm{M}$ protein in immunofixation electrophoresis; (5) refsum disease: it is a genetic problem of motor and sensory peripheral neuropathy caused by the phytanic oxidase deficiency, which leads to deposition of phytanic acid. This is usually found in adolescents and adults with symptoms as peripheral neuropathy, ataxia, deafness, retinitis pigmentosa, scaling skins, etc. There is also significant increase of CSF protein, which leads to misdiagnosis of CIDP. The critical diagnostic criterion is the significant elevation of plasma level of phytanic acid. ${ }^{[2,6]}$

The diagnosis or confirmation of CIDP needs to be differential with other factors leading to chronic polyneuropathy, such as metabolism, drug interaction, toxicology, and connective tissue diseases. For adolescent patients, it is essential to eliminate the opportunities of different hereditary demyelinating peripheral neuropathy, such as Charcot-Marie-Tooth disease.

\section{TREATMENT}

\section{Immunotherapy}

(1) Glucocorticoid: this is usually the primary mediation for CIDP. For instance, intravenous administration of methylprednisolone (500-1,000 mg/day) for 3 to 5 consecutive days will be prescribed, followed by a gradual reduction of dose or oral administration of prednisone (morning) for 1-2 months, which will also be reduced in dose accordingly. Alternatively, intravenous administration of dexamethasone (10-20 mg/day) for 7 consecutive days will also be prescribed, shifted to $1 \mathrm{mg} / \mathrm{kg}$ prednisone (morning). After 1 to 2 months of treatment, dose could be reduced or changed to oral administration of prednisone (1 $\mathrm{mg} / \mathrm{kg}$ for morning) for 1 to 2 months followed by a gradual reduction of dose. The mentioned oral therapy by prednisone could be reduced to $5-10 \mathrm{mg}$ and last for more than 6 months; afterward, the treatment could be terminated according to the conditions. During the glucocorticoid therapy, supplementary calcium and potassium, and gastric mucosa protected may need to be considered; (2) intravenous immunoglobulin (IVIg): the treatment course consists of intravenous perfusion of $400 \mathrm{mg} / \mathrm{kg}$ $\lg$ for 3 to 5 days, which is repeated once in a month for 3 months. If necessary, the treatment could be extended to months; (3) plasma exchange: for CIDP patients who are applicable with, plasma could be exchanged for $30 \mathrm{~mL} / \mathrm{kg}$ each time. The course, once a month only, consists of 3 to 5 exchanges, each of which separated by 2 to 3 days. Cautiously, plasma exchange is only allowed 3 weeks after the treatment of IVIg; (4) other immunosuppressants: when the aforementioned approaches failed, or in condition of hormonal dependence or intolerance, other immunosuppressants, such as azathioprine, CTX, cyclosporine and methotrexate, could be considered. Azathioprine, clinically common for CIDP, could be prescribed in $1-3 \mathrm{mg} / \mathrm{kg}$, orally administered in $2-3$ times, with also the monitor of hepatic and renal functions and blood biochemistry. ${ }^{[5,7-9]}$ 
Neurotrophic approaches

Mainly vitamin B therapy: B1, B12 (methylcobalamin, adenosine cobalamin), B6, etc. could be included.

\section{Symptomatic treatment}

For neuropathic pain, carbamazepine, amitriptyline, tramadol, gabapentin and pregabalin, etc. could be prescribed.

\section{Rehabilitation}

When the CIDP condition is under controlled, early start of exercises of neurological rehabilitation can prevent muscle atrophy and contractures.

\section{PROGNOSIS}

The prognosis in remission relapsing type of CIDP is better than the chronic progressive type; $70-90 \%$ of patients can be improved well by immunotherapy, but not in a minor group. In some rare cases, immunotherapy is potent in treatment for a transient period, following by the dependence.

The Chinese version of this guideline has been published in Chin J Neurol 2010;43:586-8.

\section{Acknowledgments}

The authors sincerely thank Prof. Shu-You Fang, Yu-Pu Guo, De-Xuan Kang, Pei-Gen Kuang, XiuLing Liang, Zhuo-Lin Liu, Ming-Li Rao, Ding-Guo Shen, Xiao-Fu Tang, Li-Juan Wu for the help in the preparation of this guideline and recommendations.

\section{Financial support and sponsorship}

None.

\section{Conflicts of interest}

There are no conflicts of interest.

\section{Patient consent \\ There is no patient involved.}

\section{Ethics approval}

This article does not contain any studies with human participants or animals.

\section{REFERENCES}

1. French CIDP Study Group. Recommendations on diagnostic strategies for chronic inflammatory demyelinating polyradiculoneuropathy. $J$ Neurol Neurosurg Psychiatry 2008;79:115-8.

2. Said G. Chronic inflammatory demyelinating polyneuropathy. Neuromuscul Disord 2006;16:293-303.

3. Koski CL, Baumgarten M, Magder LS, Barohn RJ, Goldstein J, Graves M, Gorson K, Hahn AF, Hughes RA, Katz J, Lewis RA, Parry GJ, van Doorn P, Cornblath DR. Derivation and validation of diagnostic criteria for chronic inflammatory demyelinating polyneuropathy. $J$ Neurol Sci 2009;277:1-8.

4. Dyck PJ, Thomas PK. Peripheral Neuropathy. 4th ed. Philadelphia: Elsevier Saunders; 2005.

5. Hughes RA, Bouche P, Cornblath DR, Evers E, Hadden RD, Hahn A, Illa I, Koski CL, Léger JM, Nobile-Orazio E, Pollard J, Sommer C, Van den Bergh P, van Doorn PA, van Schaik IN. European Federation of Neurological Societies/Peripheral Nerve Society guideline on management of chronic inflammatory demyelinating polyradiculoneuropathy: report of a joint task force of the European Federation of Neurological Societies and the Peripheral Nerve Society. Eur J Neurol 2006;13:326-32.

6. Feldman EL, Grisold W, Russell JW, Löscher WN. Atlas of Neuromuscular Diseases - A Practical Guideline. New York: Springer; 2004.

7. Köller H, Schroeter M, Kieseier BC, Hartung HP. Chronic inflammatory demyelinating polyneuropathy -- update on pathogenesis, diagnostic criteria and therapy. Curr Opin Neurol 2005;18:273-8.

8. Elovaara I, Apostolski S, van Doom P, Gilhus NE, Hietaharju A, Honkaniemi J, van Schaik IN, Scolding N, Soelberg Sørensen P, Udd B. EFNS guidelines for the use of intravenous immunoglobulin in treatment of neurological diseases: EFNS task force on the use of intravenous immunoglobulin in treatment of neurological diseases. Eur J Neurol 2008;15:893-908.

9. Ropper AH, Brown RH. Adams and Victor's principles of neurology. 8th ed. Columbus: McGraw-Hill Professional; 2005. 